www.conferenceie.ase.ro

\title{
AN OPTIMIZATION MODEL FOR SENSOR PROCUREMENT IN ELDERLY MONITORING
}

\author{
Constanţa Zoie RĂDULESCU \\ National Institute for Research and Development in Informatics \\ zoie.radulescu@ici.ro \\ Delia Mihaela RĂDULESCU \\ National Institute for Research and Development in Informatics \\ delia.radulescu@ici.ro \\ Mihaela TOMESCU \\ National Institute for Research and Development in Informatics \\ mihaela.tomescu@ici.ro
}

\begin{abstract}
The rapid increase in the number of elderly population is forcing rapid rises in healthcare costs. The development of wireless communication technologies, sensing technologies, embedded systems and nanotechnologies makes it possible to develop systems that monitor activities of elderly continuously. Sensors or collections of sensors, forming sensor architectures constitute an important fraction of the cost of an elderly monitoring solution. However, the compromise between the cost of procurement and the performance of a set of sensors is not yet well studied. In the paper, we have developed an optimization model for sensors procurement in elderly monitoring. This model considers the types of sensors for monitoring the elderly, the cost of these sensors provided by different suppliers, the performance assessed by various criteria and the amount of money available for their purchase. This model aims at aiding health management decisions. For validation the optimization model is applied to a case study.
\end{abstract}

Keywords: elderly, health sensors, optimization model, sensor procurement.

JEL classification: C6

DOI: $10.12948 / \mathrm{ie} 2019.07 .01$

\section{Introduction}

Population ageing is a long-term trend which began several decades ago in Europe. In an attempt to look at future trends for population ageing, Eurostat's latest set of population projections (EUROPOP2015) were made covering the period from 2015 to 2080. In EUROPOP2015 the EU-28's population is projected to increase to a peak of 528.6 million around 2050 and thereafter gradually decline to 518.8 million by 2080 [1]. This shift towards increasing the number of older people is important challenges for countries because they need to ensure that social and health systems are aligned with the needs of the elderly.

The proactive approach, characterized by early detection of a negative health condition, prevention and management of long-term care, leads to a restructuring of the elderly health care systems [2].The environment assisted living (AAL) paradigm is very important today, because it aims to develop innovative concepts or integrate innovative technologies, products, systems or services that can contribute to a better and safer life. The general goal of AAL solutions is to apply the Ambient Intelligence (AmI) concept and technologies to enable elderly with specific demands to live longer in their natural environment [3]. 
Many health applications and sensors (electrocardiogram sensors, blood pressure sensors, blood glucose meters, etc.) have been developed, which are used by patients for monitoring and controlling their health. These applications and sensors produce personal health data that can be used for recommendations and treatment purposes [4]. Monitoring data of patients gathered by heterogeneous sensors play an essential role in AAL. Various AAL stakeholders own or have access to different sensors and monitored data.

The activity of elderly monitoring is a very active area of research. There are many parts which are to be considered in a global way [5]: (1) types of sensors to be used; (2) type of wireless protocols to be employed; (3) monitoring of activities to be considered; (4) methodology to determine activities or extraction of important features; (5) design and development of small, light-weight, powerful and low-cost smart sensor nodes; (6) harvesting of energy for normal operation and communication; (7) ability to be used with the present day mobile devices; (8) flexible to configure the system for a new user without much difficulty.

An important aspect is the compromise between the cost of procurement and the performance of a set of sensors used in elderly monitoring. Many different sensors are available to manufacturers and knowledge about the application type and basic operation conditioning requirements can create a more informed and accurate choice.

The issue of supplier selection has become a popular problem, being extensively studied in recent years [6], [7]. Chai et al. [8] classified methodologies employed to select suppliers into three, Multi-criteria Decision-making MCDM techniques, Mathematical Programming MP models, and Artificial Intelligence AI approaches.

In section 2 of the paper a description of types of sensors for health in elderly monitoring is presented. These sensors are in principal wearable sensors and ambient sensors. In section 3 is detailed our optimization model for sensor procurement in elderly monitoring. This model considers the types of sensors for monitoring the elderly, the cost of these sensors provided by different suppliers, the performance assessed by various criteria and the amount of money available for their purchase. In section 4 the optimization model is applied to a case study. We present conclusions in section 5 and focus on the optimization model.

\section{Types of sensors for health elderly monitoring}

The important sensors for health information in elderly monitoring are wearable sensors and ambient sensors.

\subsection{Wearable Sensors}

The development of innovative wearable technologies has raised great interest in new means of data collection in healthcare and biopharmaceutical research and development. Multiple applications for wearable's sensors have been identified in a number of therapeutic areas. Recent advances in telecommunications, microelectronics, sensor manufacturing and data analysis techniques have opened up new possibilities for using wearable technology in the digital health systems to achieve a range of health outcomes [9]. Wearable sensors allow continuous physiological monitoring with reduced manual intervention and at low cost. These sensors can be integrated into various accessories such as garments, hats, wrist bands, socks, shoes, eyeglasses and other devices such as smart watches, head phones and smart phones. Some sensors, mostly medical-grade ones, are used on a stand-alone basis. Some smart phones offer health and fitness applications that help users to monitor their daily activities and health (e.g. diet and nutrition calories, vital signs, fitness progress, etc.).

Some monitoring systems require the gathered sensor and wearable's data to be uploaded to a remote site such as a hospital server for further clinical analysis. With the advent of cloud- 
computing, many wearable sensor systems can now be easily upgraded without the need for user installation of software in their monitoring devices, which makes it easier and cheaper to maintain the health monitoring system networks [10].Some examples of categories of wearable sensors are: Inertial (Accelerometer, Gyroscopes, Pressure sensors, Magnetic field sensors), Location (GPS), Medical (Blood pressure cuff, Electrocardiogram (ECG), Heart Rate), Physiological (Spirometer, Electrooculography, Electrodermal activity, Galvanic skin response), Image (SenseCam), Actigraphy, etc.

\subsection{Ambient sensors}

Ambient intelligence makes use of sensors in the ambient space of smart homes to automatically recognize certain activities or conditions that occur. The goal of ambient intelligence is to detect not only the identities and locations of the occupant of the smart space, but also the detailed activities of elderly.

The concept of ambient sensors is closely linked with the smart home, in which various types of sensors/devices are integrated into everyday objects. Infrastructure in the smart home is connected by network technologies. Via the sensors the information refers to vital signs and behavioral information of the elderly. Machine vision is one of the most common approaches for elderly monitoring in smart homes. However, other sensors (e.g., thermometer, hygrometer, window contact, door contact, light switch, remote control switch, floor vibration sensors, etc.) are also used for elderly health and behavior monitoring.

Some examples of categories of wearable sensors are: Environmental sensors (Thermometer, Hygrometer, Window contact, Door contact, Light switch, Remote control switch), Location (Infra-red, Zigbee, Active RFID), Activity Detection, Sleep Monitoring, home automation, etc.

\section{An optimization model for sensor procurement in elderly monitoring}

A healthcare institution needs to buy several sensors of various types for elderly monitoring. There is an offer from several suppliers on the market for various types of health sensors. The problem is to find a set of suppliers that maximizes the overall performance.

In the following we shall formulate an optimization model for sensor procurement in elderly monitoring. The model input data are presented in Table 1.

Table 1. The model input data

\begin{tabular}{|l|c|l|}
\hline Nr.crt. & Input data & Description \\
\hline 1 & $m$ & Number of types of sensors \\
\hline 2 & $n$ & Number of suppliers \\
\hline 3 & $d_{i j}$ & Upper bound for the number of sensors of type $i$ sold by supplier $j$ \\
\hline 4 & $c_{i j}$ & Cost of one sensor of type $i$ from supplier $j$ \\
\hline 5 & $p_{i j}$ & Performance of one sensor of type $i$ from supplier $j$ \\
\hline 6 & $M$ & Budget available for buying sensors \\
\hline 7 & $a_{i}$ & Lower bound for the number of sensors of type $i$ \\
\hline 8 & $b_{i}$ & Upper bound for the number of sensors of type $i$ \\
\hline
\end{tabular}

The decision variable is the matrix $X=\left(x_{i j}\right)$ where $x_{i j}=$ the number of sensors of type $i$ bought from supplier $j$. For the construction of matrix $P=\left(p_{i j}\right)$ of sensors performance, a group of experts take into consideration multiple characteristics of sensors and quality criteria. Some 
www.conferenceie.ase.ro

examples for sensors characteristics are: Range, Resolution, Sensitivity, Error, Accuracy, Precision, Impedance, Deadband, Response time, Bandwidth, Operating temperature. Some examples for sensors criteria are: Dynamic range, Required resolution and sensitivity, Required accuracy and precision, Power available for sensing, Availability, Cost, Ease of use, Ease of maintenance.

The performance maximization model for the sensors procurement

The objective function is the overall performance of the sensors. The first constraint states that the total number of sensors type $i$ is in range $\left[a_{i}, b_{i}\right], i=1,2, \ldots, m$.

The second constraint states that the total cost is equal or lower than the available budget for buying sensors.

The last constraint states that the number of sensors of type $i$ bought from supplier $j$ is equal or lower than the upper bound for the number of sensors of type $i$ sold by supplier $j$.

$$
\left\{\begin{array}{l}
\max \left(\sum_{i=1}^{m} \sum_{j=1}^{n} p_{i j} x_{i j}\right) \\
a_{i} \leq \sum_{j=1}^{n} x_{i j} \leq b_{i}, \quad i=1,2, \ldots, m \\
\sum_{i=1}^{m} \sum_{j=1}^{n} c_{i j} x_{i j} \leq M \\
x_{i j} \leq d_{i j}, \quad i=1,2, \ldots m, j=1,2, \ldots, n
\end{array}\right.
$$

Establishing the range for the budget parameter $M$.

In order to obtain the lower bound of budget (parameter $M_{l}$ ) the following optimization problem is solved:

$$
\left\{\begin{array}{l}
\min \left(\sum_{i=1}^{m} \sum_{j=1}^{n} c_{i j} x_{i j}\right) \\
a_{i} \leq \sum_{j=1}^{n} x_{i j} \leq b_{i}, i=1,2, \ldots m \\
x_{i j} \leq d_{i j}, i=1,2, \ldots m, j=1,2, \ldots, n
\end{array}\right.
$$

Optimal value of the above problem is the parameter $M_{1}$

In order to obtain the upper bound for the budget (parameter $M_{2}$ ) the following optimization problem is solved:

$$
\left\{\begin{array}{l}
\max \left(\sum_{i=1}^{m} \sum_{j=1}^{n} c_{i j} x_{i j}\right) \\
a_{i} \leq \sum_{j=1}^{n} x_{i j} \leq b_{i}, \quad i=1,2, \ldots, m \\
x_{i j} \leq d_{i j}, \quad i=1,2, \ldots m, j=1,2, \ldots, n
\end{array}\right.
$$

The optimal value of the above problem is the parameter $M_{2}$. The range of parameter $M$ is $\left[M_{1}, M_{2}\right]$.

\section{Case study}

Suppose that a manager of a healthcare institution wants to buy 7 types of sensors (denoted with T1, T2, T3, T4, T5, T6 and T7) for elderly monitoring. In the market there are 5 suppliers (denoted with F1, F2, F3, F4, F5) that sell the seven types of sensors.

The performance of each type of sensor is evaluated across several criteria. Lower bound and upper bound for the number of sensors is fixed. The matrix of types of sensors costs are build. 
One can compute the lower and the upper limit for the budget $M$. In order to obtain the lower bound of budget (parameter $M_{l}$ ) the optimization problem (2) is solved. The optimal value of the objective function is $M_{1}=5948$.

In order to obtain the upper bound of budget (parameter $M_{2}$ ) the optimization problem (3) is solved. The optimal value of the objective function is $M_{2}=13935$. The range for the parameter $M$ is $\left[M_{1}, M_{2}\right]=[5948,13935]$.

We search, for several values of $M$ in the range [5948, 13935], the optimal solution by solving the model (1). We consider the step length $h=(M 2-M 1) / q$ and $S_{j}=M_{1}+j h, j=0,1, \ldots, q$ and solve the model (1) for each value of $M$ in the set $\left\{S_{0}, S_{1}, \ldots, S_{q}\right\}_{\text {which is included in the range }}$ [5948, 13935]. For each $M=S_{j}$ we will compute the optimal value of the objective function.

The efficient frontier corresponding to the $q$ optimal solutions is shown in Figure 1. On the $\mathrm{x}$ axis is the parameter $M$ that varies in the range [5948, 13935] and on the y axis is the optimal value of the calculated objective function. In Table 2 is presented the efficient frontier of the maximization performance for $q=11$ values of $M$. For each value of $M$ the optimal solution is presented in the corresponding column.

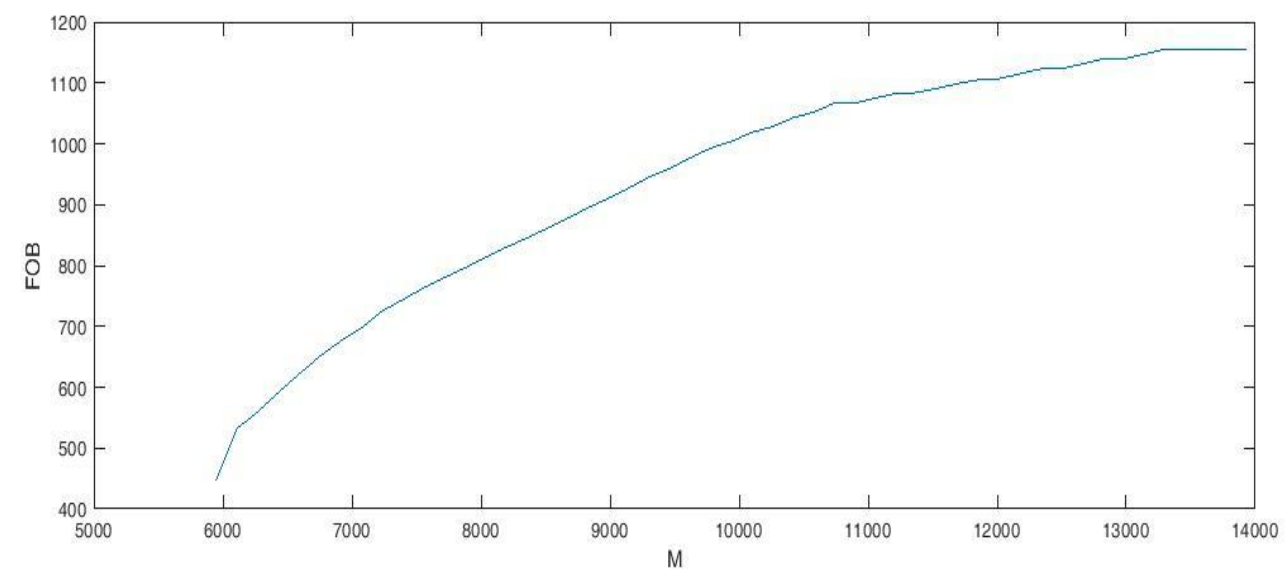

Figure 1. Efficient Frontier

Table 2.Optimal solutions for several values of parameter $M$

\begin{tabular}{|c|c|c|c|c|c|c|c|c|c|c|c|c|}
\hline \multirow{2}{*}{$\begin{array}{l}\text { Sensor } \\
\text { type }\end{array}$} & \multirow[b]{2}{*}{ Suppliers } & \multicolumn{11}{|c|}{ Solutions (columns) for various values of $M$} \\
\hline & & $\begin{array}{l}M= \\
447\end{array}$ & $\begin{array}{l}M= \\
621\end{array}$ & $\begin{array}{l}M= \\
743\end{array}$ & $\begin{array}{l}M= \\
829\end{array}$ & $\begin{array}{l}M= \\
910\end{array}$ & $\begin{array}{l}M= \\
993\end{array}$ & $\begin{array}{r}M= \\
1052\end{array}$ & $\begin{array}{r}M= \\
1084\end{array}$ & $\begin{array}{r}M= \\
1115\end{array}$ & $\begin{array}{r}M= \\
1139\end{array}$ & $\begin{array}{r}M= \\
1155\end{array}$ \\
\hline \multirow{3}{*}{$\mathrm{T} 1$} & F2 & 0 & 9 & 19 & 20 & 19 & 20 & 19 & 19 & 20 & 20 & 0 \\
\hline & F3 & 9 & 0 & 0 & 0 & 0 & 0 & 0 & 0 & 0 & 0 & 0 \\
\hline & F5 & 0 & 0 & 0 & 0 & 0 & 0 & 0 & 0 & 0 & 0 & 20 \\
\hline \multirow{2}{*}{$\mathrm{T} 2$} & F2 & 0 & 10 & 10 & 19 & 30 & 30 & 30 & 30 & 30 & 30 & 30 \\
\hline & F5 & 10 & 0 & 0 & 0 & 0 & 0 & 0 & 0 & 0 & 0 & 0 \\
\hline \multirow{2}{*}{$\mathrm{T} 3$} & F1 & 0 & 8 & 8 & 8 & 8 & 8 & 14 & 15 & 15 & 15 & 15 \\
\hline & F2 & 8 & 0 & 0 & 0 & 0 & 0 & 0 & 0 & 0 & 0 & 0 \\
\hline \multirow{2}{*}{$\mathrm{T} 4$} & F3 & 0 & 27 & 30 & 30 & 30 & 30 & 30 & 30 & 30 & 30 & 30 \\
\hline & F4 & 15 & 0 & 0 & 0 & 0 & 0 & 0 & 0 & 0 & 0 & 0 \\
\hline \multirow{2}{*}{ T5 } & F2 & 0 & 1 & 10 & 11 & 11 & 13 & 15 & 15 & 15 & 15 & 15 \\
\hline & F5 & 9 & 8 & 0 & 0 & 0 & 0 & 0 & 0 & 0 & 0 & 0 \\
\hline \multirow{2}{*}{ T6 } & F2 & 0 & 1 & 10 & 10 & 10 & 15 & 15 & 15 & 15 & 15 & 15 \\
\hline & F4 & 10 & 9 & 0 & 0 & 0 & 0 & 0 & 0 & 0 & 0 & 0 \\
\hline
\end{tabular}


www.conferenceie.ase.ro

\begin{tabular}{|l|l|l|l|l|l|l|l|l|l|l|l|l|r|r|r|r|r|}
\hline \multirow{2}{*}{ T7 } & F1 & 9 & 9 & 8 & 9 & 9 & 0 & 0 & 0 & 0 & 0 & 0 \\
\hline & F4 & 0 & 0 & 1 & 0 & 0 & 9 & 9 & 12 & 15 & 18 & 20 \\
\hline
\end{tabular}

The optimization problems are solved with solver MINLP in GAMS.

\section{Conclusions}

We proposed an optimization model for sensors procurement in elderly monitoring that integrates sensors performance and costs. Sensor selection refers to the problem of intelligently selecting a small subset from a collection of available sensors in order to reduce the cost and maximize the performances. The optimization models (2) and (3) provide, for a specific problem, a range for the budget parameter $M$. This optimization model can be used in management decisions for buying wearable or ambient sensors for elderly monitoring.

\section{Acknowledgment}

The research presented in this paper is supported by the project "vINCI: Clinically-validated INtegrated Support for Assistive Care and Lifestyle Improvement: the Human Link" (funded by EUAAL2017 Programme and Executive Unit for Financing Higher Education, Research, Development and Innovation-UEFISCDI Romania) and by the project "Non-invasive monitoring and health assessment of the elderly in a smart environment (RO-Smart Ageing)" (funded by the Romanian Core Program of the Ministry of Research and Innovation).

\section{References}

[1] *** Population structure and ageing, https://ec.europa.eu/eurostat/statisticsexplained/index.php/Population_structure_and_ageing.

[2] A. Alexandru and M. Ianculescu, "Enabling Assistive Technologies to Shape the Future of the Intensive Senior-Centred Care: A Case Study Approach," Studies in Informatics and Control, vol. 26 (3), pp.461- 469, 2017.

[3] N. Garcia and J.P. Rodrigues, Ambient Assisted Living, Taylor \& Francis, 2015.

[4] R.A. Voicu, C. Dobre, L. Bajenaru and R.I. Ciobanu, "Human Physical Activity Recognition Using Smartphone Sensors," Sensors, vol. 19(3), pp. 458, 1-19, 2019.

[5]S.C. Mukhopadhyay, "Wearable Sensors for Human Activity Monitoring: A Review," Sensors Journal, IEEE, Vol. 15, pp. 1321-1330, 2015.

[6] J. Luan, Z. Yao, F. Zhao and X. Song, "A novel method to solve supplier selection problem: Hybridalgorithm of genetic algorithm and ant colony optimization," Mathematics and Computers in Simulation, vol. 156, pp. 294-309, 2019.

[7] M. Radulescu and C.Z. Radulescu, "A Portfolio Theory Approach to Software Vendor Selection," Studies in Informatics and Control, vol. 24 (4), pp. 379-386, 2015.

[8] J.Y. Chai, J.N.K. Liu and E.W.T. Ngai, "Application of decision-making techniques in supplier selection: a systematic review of literature," Expert System Application, vol. 40, pp. 3872-3885, 2013.

[9] E. Izmailova, J.A. Wagner and E.D. Peraklis, "Wearable Devices in Clinical Trials: Hype and Hypothesis," Clinical pharmacology and therapeutics, vol. 104(1), pp.42-52, 2018.

[10] S. Patel, H. Park, P. Bonato, L. Chan and M. Rodgers, "A review of wearable sensors and systems with application in rehabilitation,". Journal of Neuro Engineering and Rehabilitation, vol. 9(1), pp. 9-21, 2012. 